\title{
bmo-miR-0001 and bmo-miR-0015 down-regulate expression of Bombyx mori fibroin light chain gene in vitro*
}

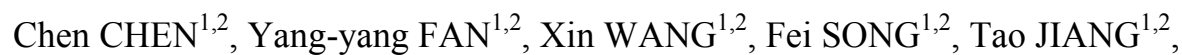 \\ Ping QIAN ${ }^{1,2}$, Shun-ming TANG ${ }^{1,2}$, Xing-jia SHEN ${ }^{\dagger 11,2}$ \\ ( ${ }^{1}$ Jiangsu Key Laboratory of Sericultural Biology and Biotechnology, School of Biotechnology, \\ Jiangsu University of Science and Technology, Zhenjiang 212003, China) \\ ( ${ }^{2}$ Key Laboratory of Silkworm and Mulberry Genetic Improvement, Ministry of Agriculture, \\ Sericultural Research Institute, Chinese Academy of Agricultural Sciences, Zhenjiang 212018, China) \\ †E-mail: shenxjsri@163.com \\ Received Apr. 6, 2015; Revision accepted Nov. 7, 2015; Crosschecked Jan. 13, 2016
}

\begin{abstract}
Based on bioinformatic analysis, we selected two novel microRNAs (miRNAs), bmo-miR-0001 and bmomiR-0015, from high-throughput sequencing of the Bombyx mori larval posterior silk gland (PSG). Firstly, we examined the expression of bmo-miR-0001 and bmo-miR-0015 in 12 different tissues of the 5th instar Day-3 larvae of the silkworm. The results showed that the expression levels of both bmo-miR-0001 and bmo-miR-0015 were obviously higher in the PSG than in other tissues, implying there is a spatio-temporal condition for bmo-miR-0001 and bmo-miR-0015 to regulate the expression of BmFib-L. To test this hypothesis, we constructed pri-bmo-miR-0001 expressing the plasmid pcDNA3.0 [ie1-egfp-pri-bmo-miR-0001-SV40] and pri-bmo-miR-0015 expressing the plasmid pcDNA3.0 [ie1-egfp-pribmo-miR-0015-SV40]. Finally, the BmN cells were harvested and luciferase activity was detected. The results showed that luciferase activity was reduced significantly $(P<0.05)$ in BmN cells co-transfected by pcDNA3.0 [ie1-egfp-pri-bmomiR-0001-SV40] or pcDNA3.0 [ie1-egfp-pri-bmo-miR-0015-SV40] with pGL3.0 [A3-luc-Fib-L-3'UTR-SV40], suggesting that both bmo-miR-0001 and bmo-miR-0015 can down-regulate the expression of BmFib-L in vitro.
\end{abstract}

Key words: Bombyx mori, MicroRNA, bmo-miR-0001, bmo-miR-0015, BmFib-L, Regulation of expression http://dx.doi.org/10.1631/jzus.B1500078

CLC number: S881.26

\section{Introduction}

MicroRNAs (miRNAs) are small non-coding RNA molecules about 19 to 22 nucleotides long, which mostly inhibit the expression or translation of target genes by complementary base pairing to the target mRNA 3' untranslated region (3'UTR) (Lee et al., 2004). They are involved in a lot of biological processes, including development, hematopoietic

\footnotetext{
¿ Corresponding author

* Project supported by the National Natural Science Foundation of China (Nos. 31172266/C1703 and 31402143/C1703)

(iD) ORCID: Chen CHEN, http://orcid.org/0000-0001-6637-3086

(C) Zhejiang University and Springer-Verlag Berlin Heidelberg 2016
}

lineage differentiation, organ formation, proliferation, apoptosis, host viral interactions, and tumorigenesis (Lim et al., 2003; Poy et al., 2004; Bushati and Cohen, 2007; Yuan et al., 2014). Research on miRNAs has been conducted on a range of organisms including animals (Dai et al., 2014; Lu et al., 2014), plants (Mallory and Vaucheret, 2006), microbes (Liu et al., 2016), and viruses (Jopling et al., 2005). Studies on miRNAs will provide a theoretical basis for posttranscriptional regulation of genes and benefit our understanding of gene regulatory mechanisms.

The silkworm (Bombyx mori) is an insect that undergoes complete metamorphosis. It not only has the biological traits common to other insects, but also has its own characteristic properties making it an 
excellent model for the study of insect genetics and molecular biology (Xia et al., 2004; 2009). With the completion of sequencing of the silkworm genome, the study of B. mori miRNA has entered a new development stage that has led to the discovery of novel miRNAs (Yu et al., 2008; 2009; Cai et al., 2010; Yang et al., 2012), prediction of miRNA targets (He et al., 2008; Zeng et al., 2009; Huang et al., 2012; Chen et al., 2013), and identification and expression of miRNAs (Zhang et al., 2009; Liu et al., 2010; Li et al., 2014). The mechanism of silk protein biosynthesis has been a popular focus of research among a large numbers of studies.

The silk glands, a special organ in the silkworm that synthesizes and secretes the silk protein, can be divided into the anterior silk gland (ASG), middle silk gland (MSG), and posterior silk gland (PSG) according to their morphological specificity and function (Inoue et al., 2000). The ASG is a catheter of silk protein with no function in silk protein biosynthesis and the MSG mainly synthesizes and secretes sericin proteins, whereas the PSG synthesizes and secretes silk fibroin, which accounts for more than $70 \%$ of the total silk protein. Fibroin comprises the fibroin heavy chain protein $(\mathrm{Fib}-\mathrm{H})$, fibroin light chain protein (Fib-L), and P25/fibrohexamerin protein (P25) (Sprague, 1975; Yamaguchi et al., 1989). Studies have shown that B. mori miRNAs are involved in regulation of fibroin gene expression. Four bmo-miRNAs have been predicted to have potential binding sites in the BmFib-L gene and might play important roles in the regulation of silk protein biosynthesis (Cao et al., 2008). The miRNA bmo-miR-2b also regulates the expression of BmP25 (Huang et al., 2011). Both bmo-miR-965 and bmo-miR-1926 inhibit the expression of BmFib-L in vitro (Huang et al., 2012). However, bmo-miR-2739 has been shown to upregulate the expression of BmFib-H (Song et al., 2014).

In the previous high-throughput sequencing of PSG small RNAs in our laboratory, 35 novel bmomiRNAs were obtained, among which two, bmomiR-0001 and bmo-miR-0015, are predicted to function in regulating the expression of BmFib-L. To validate this prediction, the expressions of bmo-miR0001, bmo-miR-0015, and their potential target gene $B m F i b-L$ were examined by reverse transcription polymerase chain reaction (RT-PCR) at the mRNA level. The regulatory function of these two miRNAs was also tested using a dual luciferase reporter (DLR) system in BmN cells.

\section{Materials and methods}

\subsection{Materials}

The domesticated silkworm (B. mori) used in this experiment was of the strain P50, provided by the Sericultural Research Institute, Chinese Academy of Agricultural Sciences (CAAS; Zhenjiang, China). Plasmids pcDNA3.0 (Invitrogen, Shanghai, China), pRL-CMV (containing a Renilla luciferase gene), and pGL3.0 [A3-luc-Fib-L-3'UTR-SV40], and BmN cells were preserved or constructed by the Key Laboratory of Silkworm and Mulberry Genetic Improvement, Ministry of Agriculture, China. Restriction enzymes, T4 DNA ligase, PCR reagents, and a reverse transcription kit were purchased from TaKaRa (Dalian, China). Primers were synthesized by Sangon Biotech (Shanghai, China). Fetal bovine serum (FBS) and TC-100 cell culture medium were purchased from Invitrogen. Cell perfect transfection reagent was purchased from UcallM (Wuxi, China). A DualLuciferase $^{\circledR}$ Reporter (DLR ${ }^{\mathrm{TM}}$ ) Assay System kit was purchased from Promega (Madison, USA).

\subsection{Methods}

2.2.1 Screening of $B$. mori miRNAs regulating BmFib- $L$ expression

To screen $B$. mori miRNAs for those that may regulate the expression of BmFib-L, the BmFib-L sequence from the National Center for Biotechnology Information (NCBI) (http://www.ncbi.nlm.nih.gov/gene) and sequences of B. mori mature miRNAs from Solexa sequencing were analyzed by RNAhybrid software (http://bibiserv.techfak.uni-bielefeld.de/rnahybrid). The potential targets of miRNAs were identified as having perfect sequence complementarity between the seed region of the miRNA (a 7-nucleotide sequence from bases 2 to 8 at the $5^{\prime}$ end of the miRNAs) and the 3'UTR of target (BmFib-L) mRNAs, and less than $-20.0 \mathrm{kcal} / \mathrm{mol}(1 \mathrm{kcal} / \mathrm{mol}=4.182 \mathrm{~kJ} / \mathrm{mol})$ free energy in the secondary structure of the miRNA/ mRNA duplex (Huang et al., 2010). 
2.2.2 Cloning of novel miRNAs and prediction of the secondary structures of their precursors

Total RNA was extracted from the PSG of Day-3 5 th instar larvae using TRIzol (Invitrogen) according to the manufacturer's instructions. The complementary DNAs (cDNAs) were synthesized from total RNA using miRNA specific stem-loop primers according to the method of Kramer (2011). Subsequently, PCR was carried out with the following cycling conditions: initial denaturation at $94{ }^{\circ} \mathrm{C}$ for $5 \mathrm{~min}, 34$ cycles of $94^{\circ} \mathrm{C}$ for $30 \mathrm{~s}, 55^{\circ} \mathrm{C}$ for $25 \mathrm{~s}$, and $72{ }^{\circ} \mathrm{C}$ for $30 \mathrm{~s}$, and final extension at $72{ }^{\circ} \mathrm{C}$ for $10 \mathrm{~min}$. The PCR products were separated by electrophoresis in $4 \%(0.04 \mathrm{~g} / \mathrm{ml})$ agarose gels. After purification, the gene fragments were cloned into a pMD18-T vector and sequenced. Using ClustalX 1.83, multiple sequence alignment software was applied for further confirmation of sequencing, and secondary structures were predicted using RNAfold (http://rna.tbi.univie. ac.at/cgi-bin/RNAfold.cgi). The sequences of the primers are listed in Table 1.

\subsubsection{Detection of expression by RT-PCR}

To investigate the possibility of spatio-temporal effects on the predicted function of bmo-miRNAs in regulating the expression of $B m F i b-L$, the expression levels of potential bmo-miRNAs and $B m F i b-L$ in the silk gland of the silkworm larvae were analyzed. Firstly, total RNAs were extracted from different tissues including the head, epidermis, fat body, Malpighian tubule, ASG, MSG, PSG, trachea, testis, ovary, midgut, and haemolymph of the 5 th instar Day-3 larva using TRIzol (Invitrogen) according to the manufacturer's instructions. cDNAs were synthesized from total RNAs using a cDNA reverse transcription kit (TaKaRa). Subsequently, PCR was carried out and the products were detected as described above and photographed under UV light. Three parallel experiments were carried out and the $B$. mori U6 gene served as an internal control. Finally, the relative expression levels of the potential bmo-miRNAs and $B m F i b-L$ in different tissues were determined using Gel-Pro Analyzer software (Media Cybernetics, USA).

\subsubsection{Construction of expression vectors}

To validate the function of potential bmomiRNAs in the regulation of $B m F i b-L$ expression in vitro, bmo-miRNA expression vectors were con- structed as described previously (Huang et al., 2011). The luciferase gene (luc) fused BmFib-L 3'UTR expression vectors pGL3.0 [A3-luc-Fib-L-3'UTR-SV40] constructed previously in our laboratory (Huang et al., 2012) were used. The nucleotide sequences of precursors of the potential bmo-miRNAs and their upand down-stream flanking regions were downloaded from SilkBase (http://silkbase.ab.a.u-tokyo.ac.jp/cgi-bin/ index.cgi). Then primers designed using Oligo 7 software (molecular Biology Insights, Inc., Cascade, CO, USA) were constructed for PCR of the potential bmo-miRNAs and the PCR products were cloned into the pET-18 vector for sequence determination. The pET-18-pri-miR-0001 and pET-18-pri-miR-0015 constructs were double digested with HindIII and BamHI, separately. The nucleotide sequences of the mature bmo-miRNAs, bmo-miR-0001, and bmomiR-0015, and their flanking regions $( \pm 100 \mathrm{bp})$ were cloned into the pCDNA3.0 plasmid downstream of the egfp gene controlled by the $B$. mori nucleopolyhedrovirus (BmNPV) iel promoter for construction of the bmo-miRNA expression vectors pcDNA3.0 [ie1-egfp-pri-bmo-miR-0001-SV40] and [ie1-egfppri-bmo-miR-0015-SV40]. The structure of these constructs was confirmed by sequencing (Sangon Biotech).

2.2.5 Cell culture, transfection, and transient expression assay

The method of Zhou et al. (2002) was used for routine $\mathrm{BmN}$ cell culture. Cells were seeded into 12 -well plates $(600 \mu \mathrm{l}$ for each) at a density of about $5 \times 10^{5}$ cells $/ \mathrm{ml}$ and cultured for $24 \mathrm{~h}$ (Song et al., 2013). Before transfection, the medium was removed and the cells were washed twice with serum-free TC-100 medium. In the treatment group, cells in each well were co-transfected with a 1.6- $\mu \mathrm{g}$ mixture of DNAs including $0.7 \mu \mathrm{g}$ pcDNA3.0 [ie1-egfp-pribmo-miR-0001-SV40] or pcDNA3.0 [ie1-egfp-pribmo-miR-0015-SV40], $0.7 \mu \mathrm{g}$ pGL3.0 [A3-luc-Fib$L-3$ 'UTR-SV40], and $0.2 \mu \mathrm{g}$ internal plasmid pRLCMV via PerFect Cellfectin reagent (UcallM), according to the manufacturer's manual (PerFect; UcallM). In the control group, cells in each well were co-transfected with $1.6 \mu \mathrm{g}$ of a mixture DNAs including $0.7 \mu \mathrm{g}$ pcDNA3.0 [iel-egfp-SV40], $0.7 \mu \mathrm{g}$ pGL3.0 [A3-luc-Fib-L-3'UTR-SV40], and $0.2 \mu \mathrm{g}$ pRL-CMV. There were three replicates for each experiment. 
Before harvesting, cells were observed under an inverted fluorescence microscope. At $72 \mathrm{~h}$ post transfection (hpt) cells were harvested by centrifugation at $9000 \mathrm{~g}$ for $5 \mathrm{~min}$ at $4{ }^{\circ} \mathrm{C}$, washed once in phosphate-buffered saline and lysed in $150 \mu \mathrm{l}$ of passive lysis buffer (Promega). The supernatants were used for luciferase activity assay using a Luc assay kit (Promega) in a GloMax ${ }^{\circledR}$ 20/20 Luminometer (Turner Biosystems Inc., Sunnyvale, CA, USA; $2 \mathrm{~s}$ delay and read at $10 \mathrm{~s})$ as the relative luminescence unit (RLU) (Zhao et al., 2007). The activity of firefly was normalized by the Renilla luciferase activity of pRL-CMV.

\section{Results}

\subsection{Prediction of novel $B$. mori miRNAs targeting BmFib-L 3'UTR}

Target prediction software RNAhybrid was used to assess the scores and the complementarity between the miRNA seed regions and the target site. Among 35 novel B. mori miRNAs obtained from previous Solexa sequencing (Song et al., 2015), bmo-miR0001 and bmo-miR-00015 were predicted to have the potential to target BmFib-L $3^{\prime}$ UTR. Moreover, pribmo-miR-0001 binds BmFib-L 3'UTR starting from the 43rd base, while pri-bmo-miR-0015 starts from the 5th base, suggesting that bmo-miR-0001 and bmo-miR-00015 function independently (Fig. 1).

\subsection{Cloning of candidate miRNAs and prediction of secondary structures of their precursors}

For each miRNA, RT-PCR was performed with the primers above using the total RNA of the 5th

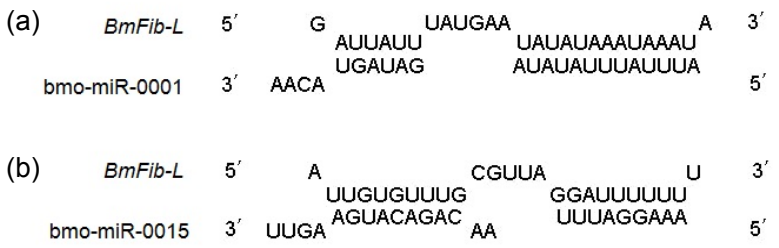

Fig. 1 Prediction of the binding sites of bmo-miR-0001 and bmo-miR-0015 in BmFib-L mRNA using RNAhybrid software

(a) Prediction of the binding site of bmo-miR-0001 in BmFib-L mRNA. The folding energy was $-16.3 \mathrm{kcal} / \mathrm{mol}$. The initiation binding site at the target gene was the 43rd base of the 3'UTR; (b) Prediction of the binding site of bmo-miR-0015 in $B m F i b-L$ mRNA. The folding energy was $-15.2 \mathrm{kcal} / \mathrm{mol}$. The initiation binding site at the target gene was the 5 th base of the 3'UTR

instar Day-3 larvae of the silkworm as a template, and a band of about $60-80 \mathrm{bp}$ was separated by agarose gel electrophoresis, consistent with expectations (Fig. 2a). Sequencing results revealed that the nucleotide sequences of the two cloned miRNAs were the same as those from Solexa sequencing (Figs. $2 b$ and 2c): bmo-miR-0001, AUU UAU UUA UAU AGA UAG UAC AA; bmo-miR-0015, AAA GGA UUU AAC AGA CAU GAA GUU. The secondary structures of their precursors were typical of miRNA precursors, with stem-loops (Fig. 3). The free energies of the bmo-miR-0001 and bmo-miR-0015 precursors were -18.10 and $-25.80 \mathrm{kcal} / \mathrm{mol}$, respectively.

\subsection{Expression of bmo-miR-0001 and bmo-miR- 0015 and their target gene BmFib-L}

The RT-PCR results showed that in the 12 tissues tested bmo-miR-0001 expression was detected only in the PSG (Fig. 4a). In contrast, bmo-miR-0015 expression was detected not only throughout the silk

Table 1 Primers used in the experiments

\begin{tabular}{lll}
\hline \multicolumn{1}{c}{ Primer } & & \multicolumn{2}{c}{ Sequence $\left(5^{\prime} \rightarrow 3^{\prime}\right)$} \\
\hline bmo-miR-0001 & RT & GTCGTATCCAGTGCAGGGTCCGAGGTATTCGCACTGGATACGACTTGTAC \\
& Forward & GCCCGATTTATTATATAGATAGT \\
& Reverse & GTGCAGGGTCCGAGGT \\
bmo-miR-0015 & RT & GTCGTATCCAGTGCAGGGTCCGAGGTATTCGCACTGGATACGACAACTTC \\
& Forward & GTGCAGGGTCCGAGGT \\
& Reverse & CCCAAGCTTAAGTCCATTCCAATAGC \\
U6 & RT & GTCGTATCCAGTGCAGGGTCCGAGGTATTCGCACTGGATACGACACG \\
& Forward & CCTGCGCAAGGATGAC \\
pri-bmo-miR-0001 & Reverse & GTGCAGGGTCCGAGGT \\
& Forward & CGGGATCCAGCTCACCTACATGTTA \\
pri-bmo-miR-0015 & Reverse & CCCAAGCTTAAGTCCATTCCAATAGC \\
& Forward & CGGGATCCACAAGTACGCAAAAGCT \\
& Reverse & CCCAAGCTTAAGGCGCACCATCAATT \\
\hline
\end{tabular}




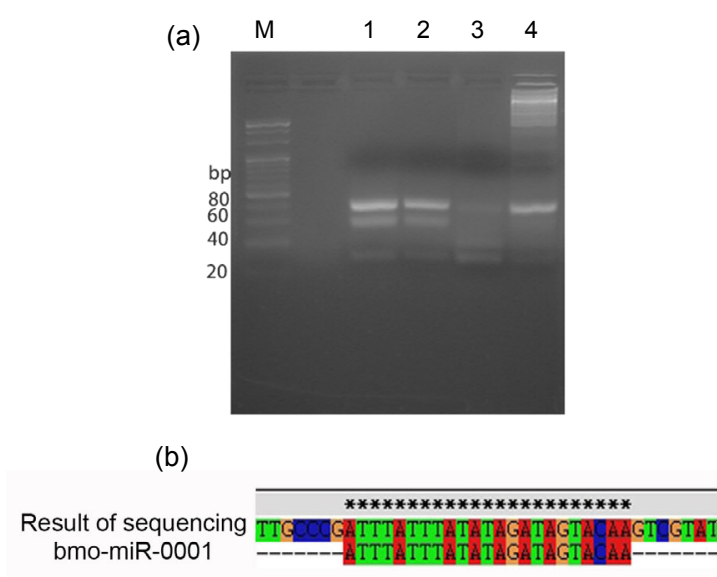

(c)

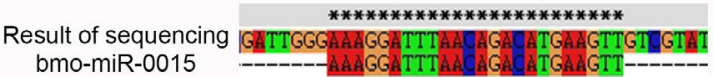

Fig. 2 Electrophoresis pattern of RT-PCR products and multiple sequence alignments of bmo-miR-0001 and bmo-miR-0015 by ClustalX 1.83

(a) Electrophoresis pattern of RT-PCR products of bmo-miR-0001 and bmo-miR-0015 (M: 20 bp marker; 1: bmo-miR-0001; 2: bmo-miR-0015; 3: bmo-miR-26* (control); 4: U6); (b) Sequence alignment result of bmomiR-0001; (c) Sequence alignment result of bmo-miR-0015

glands (ASG, MSG, and PSG), but also in the head, fat body, Malpighian tubule, testis, trachea, ovary, and midgut, but the expression level was highest in the PSG (Fig. 4b). BmFib-L expression was detected not only in the PSG, but also in the fat body, testis, MSG, trachea, and ovary (Fig. 4c). This is consistent with the results of Sun et al. (2009) and is known as "leaking expression" (Wang and $\mathrm{Lu}, 2006$ ). The above experimental results show that spatio-temporal conditions affect the regulation of the expression of BmFib-L by bmo-miR-0001 and bmo-miR-0015. Together with the target prediction result, we deduced that bmo-miR-0001 and bmo-miR-0015 may be involved in the regulation of BmFib- $L$ expression in $B$. mori larvae.

\subsection{Validating the roles of bmo-miR-0001 and bmo-} miR-0015 in regulating the expression of BmFib-L

\subsubsection{Construction of expression vectors}

The expression vectors pcDNA3.0 [ie1-egfp-pribmo-miR-0001-SV40] for miR-0001 and pcDNA3.0 [ie1-egfp-pri-bmo-miR-0015-SV40] for miR-0015 were successfully constructed to contain the BmNPV (a)

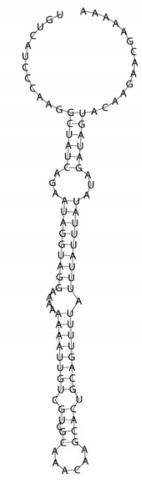

(b)

bmo-miR-0001

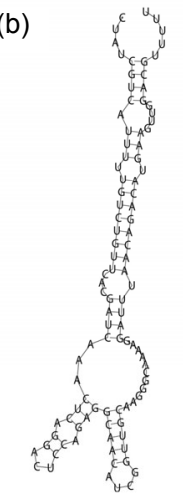

bmo-miR-0015

Fig. 3 Stem-loop structures of bmo-miR-0001 and bmo-miR-0015 predicted by RNAfold

(a) Secondary structure of the bmo-miR-0001 precursor;

(b) Secondary structure of the bmo-miR-0015 precursor

(a)

$\begin{array}{llllllllllll}1 & 2 & 3 & 4 & 5 & 6 & 7 & 8 & 9 & 10 & 11 & 12\end{array}$

(b)

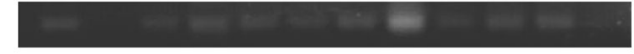

(c)

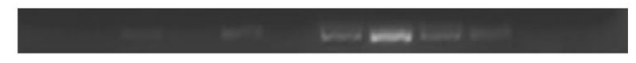

(d)

Fig. 4 Expression analyses of bmo-miR-0001, bmomiR-0015, BmFib-L, and $\mathrm{U} 6$ in different tissues of 5 th instar Day-3 larvae of $B$. mori

(a) Expression of bmo-miR-0001; (b) Expression of bmomiR-0015; (c) Expression of BmFib-L; (d) Expression of internal gene U6. 1, head; 2, epidermis; 3, fat body; 4, Malpighian tubule; 5, testis; 6, anterior silk gland; 7, middle silk gland; 8 , post silk gland; 9 , trachea; 10 , ovary; 11 , midgut; 12 , haemolymph

$i e 1$ promoter and an egfp reporter gene. The inserted gene fragments were tested by electrophoresis in agarose gels after double digestion with HindIII and BamHI (Fig. 5).

3.4.2 Regulation of BmFib-L expression by bmomiR-0001 and bmo-miR-0015 in BmN cells

In the treatment group, $\mathrm{BmN}$ cells were cotransfected with a mixture of pcDNA3.0 [ie1-egfppri-bmo-miR-0001-SV40] or pcDNA3.0 [ie1-egfppri-bmo-miR-0015-SV40], pGL3.0 [A3-luc-Fib-L3'UTR-SV40], and pRL-CMV. In the control group, $\mathrm{BmN}$ cells were co-transfected with a mixture of pcDNA3.0 [ie1-egfp-SV40], pGL3.0 [A3-luc-Fib-L3'UTR-SV40], and pRL-CMV. 

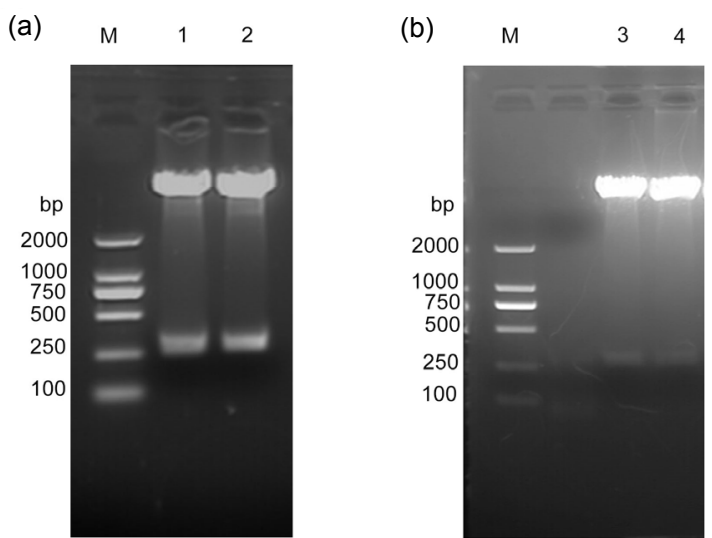

Fig. 5 Double enzyme digestion of recombinant plasmid pcDNA3.0 [ie1-egfp-pri-bmo-miR-0001-SV40] and pcDNA3.0 [ie1-egfp-pri-bmo-miR-0015-SV40] with HindIII and BamHI

(a) Identification of ie1-egfp-pri-bmo-miR-0001-SV40 structure (M: DL 2000 DNA marker; 1, 2: pcDNA3.0 [ie1egfp-pri-bmo-miR-0001-SV40] after double digestion with HindIII and BamHI); (b) Identification of ie1-egfp-pribmo-miR-0015-SV40 structure (M: DL 2000 DNA marker; 3, 4: pcDNA3.0 [ie1-egfp-pri-bmo-miR-0015-SV40] after double digestion with HindIII and BamHI)

Observations were carried out under an inverted fluorescence microscope. Results showed that recombinant plasmids had been transfected into cells and the majority of cells emitted green fluorescence (Fig. 6). Cells were then harvested and luciferase activity was assayed using a Luc assay kit (Promega) in a Luminometer 20/20, as described above. The results showed that luciferase activity in the two experimental groups decreased to $60 \%$ and $69 \%$, respectively, of that of the control group. The differences between the treatment groups and the control groups were significant $(P<0.05$; Fig. 7), indicating that bmo-miR-0001 and bmo-miR-0015 can downregulate the expression of $B m F i b-L$ in vitro.

From the above results, we conclude that BmFib-L is one of the target genes of bmo-miR-0001 and bmo-miR-0015, and that both bmo-miR-0001 and bmo-miR-0015 can down-regulate the expression of the BmFib-L gene by interacting with its 3'UTR of mRNA in vitro.

\section{Discussion}

miRNAs participate in a variety of biological activities, including cell proliferation, embryonic (a)

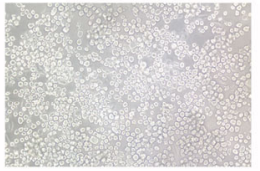

(d)

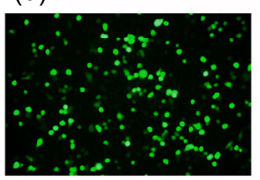

(b)

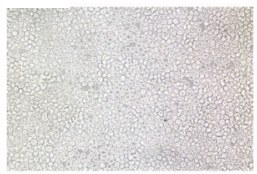

(e)

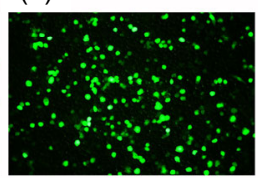

(c)

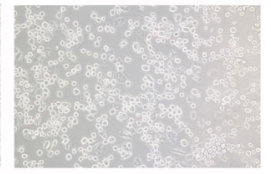

(f)

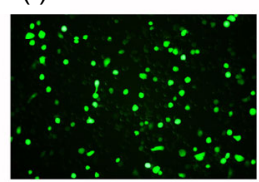

Fig. 6 Expression of enhanced green fluorescent protein in $\mathbf{B m N}$ cells transfected with recombinant plasmids (a) pcDNA3.0 [ie1-egfp-SV40]+pGL3.0 [A3-luc-Fib-L-3'UTRSV40]+pRL-CMV, bright light; (b) pcDNA3.0 [ie1-egfp-pribmo-miR-0001-SV40]+pGL3.0 [A3-luc-Fib-L-3'UTR-SV40]+ pRL-CMV, bright light; (c) pcDNA3.0 [ie1-egfp-pri-bmomiR-0015-SV40]+pGL3.0 [A3-luc-Fib-L-3'UTR-SV40]+pRLCMV, bright light; (d) pcDNA3.0 [ie1-egfp-SV40]+pGL3.0 [A3-luc-Fib-L-3'UTR-SV40]+pRL-CMV, fluorescence; (e) pcDNA3.0 [ie1-egfp-pri-bmo-miR-0001-SV40]+pGL3.0 [A3-luc-Fib-L-3'UTR-SV40]+pRL-CMV, fluorescence; (f) pcDNA3.0 [ie1-egfp-pri-bmo-miR-0015-SV40]+pGL3.0 [A3-luc-Fib-L-3'UTR-SV40]+pRL-CMV, fluorescence

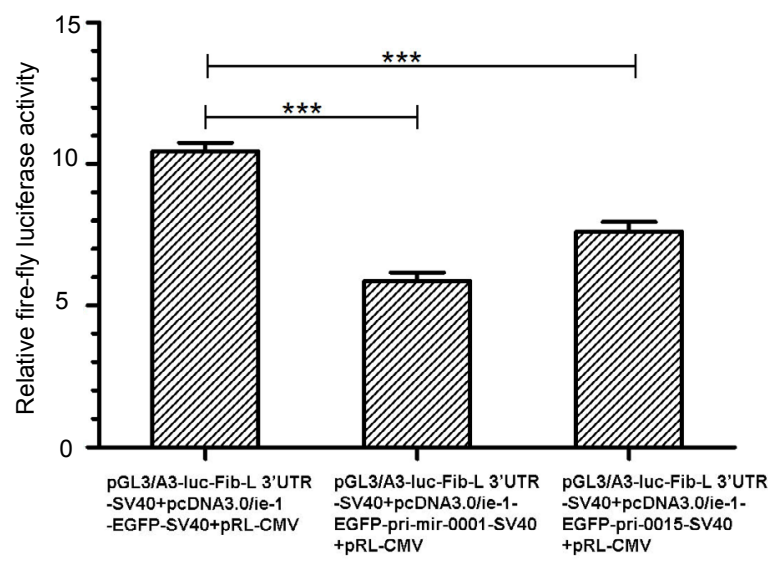

Fig. 7 Effect of bmo-miR-0001 and bmo-miR-0015 expression on luciferase activity in transfected $\mathrm{BmN}$ cells Data represented as the mean \pm standard deviation (SD) from three independent experiments. ${ }^{* * *}$ represents $P<0.001$ in unpaired $t$-tests

development, individual growth, occurrence and development of disease, and apoptosis (Johnston and Hobert, 2003; Lim et al., 2003; Poy et al., 2004). There are 563 silkworm mature miRNA sequences registered in the MiRBase (http://www.mirbase.org). Studies have shown that $B$. mori miRNAs play essential roles in silkworm development and growth. At least four bmo-miRNAs, miRNA-33, miRNA-190, 
miRNA-276, and miRNA-7, participate in the regulation of BmFib-L expression (Cao et al., 2008). miR-965 and miR-1926 have been shown to downregulate the expression of $B m F i b-L$ in vitro (Huang et al., 2012).

Based on Solexa sequencing results, we obtained two novel candidate bmo-miRNAs, bmo-miR-0001 and bmo-miR-0015, complementary to the 3'UTR of BmFib-L mRNA using RNAhybrid target prediction software. The DLR system was applied to validate the role of these two miRNAs in regulating the expression of BmFib-L in cells. For the first time, we reported that bmo-miR-0001 and bmo-miR-0015 down-regulate the expression of $B m F i b-L$ in vitro. This result is similar with that described by Huang et al. (2012) and is consistent with the finding that the majority of miRNAs inhibit expression of their target gene by binding to its 3'UTR (Fabian et al., 2010). These results will not only enrich the database of silkworm miRNAs, but also provide new experimental data for further study of miRNA function and the regulatory mechanism of silk protein biosynthesis.

B. mori silk fibroin comprises BmFib-H, BmFib-L, and BmP25 proteins (Sprague, 1975; Couble et al., 1983; Yamaguchi et al., 1989), which are produced abundantly in the silk glands of 5 th instar larvae (Wang et al., 2011). However, in this experiment bmo-miR-0001 and bmo-miR-0015 downregulated the expression of the $B m F i b-L$ gene. Recent studies have identified a competing endogenous RNA (ceRNA) gene regulatory mechanism, in which ceRNAs serve as miRNA absorbing sponges to vie with miRNAs by sharing miRNA response elements (MREs) in mRNAs (Khan et al., 2009; Ebert and Sharp, 2010). These ceRNAs act as modulators of miRNAs by lowering the available miRNA levels for the target mRNA, resulting in increased translation (Sen et al., 2014).

$\mathrm{BmN}$ cells used in this experiment were obtained from $B$. mori ovarian tissue instead of silk gland cells and might lack corresponding interference endogenous miRNAs. This may have provided an opportunity for the exogenous miRNAs (bmo-miR-0001 and bmo-miR-0015) to bind the mRNA of the target gene $(B m F i b-L)$, resulting in down-regulation of target gene expression. However, it is also highly likely that there are real differences between expression in the silkworm larvae and in the in vitro gene model.
Therefore, further study is needed on the regulation of BmFib-L expression by bmo-miR-0001 and bmomiR-0015 in vivo via over expression of silkworm miRNAs following infection by a recombinant $B$. mori baculovirus expression vector, or injection of synthetic miRNA analogues or antisense RNA of miRNAs into the 5th instar larvae.

The expression and regulation of genes producing silk protein are very complex. However, Shalgi et al. (2007) have proposed the existence of hundreds of "target hub" genes, each potentially subject to massive regulation by dozens of miRNAs. Therefore, the study of individual miRNAs is insufficient to uncover the complicated and precise regulatory mechanisms of silk protein biosynthesis. Studies of other transcription factors and more information about the regulation of silk protein biosynthesis by miRNAs are needed. Besides binding at the 3'UTR of target mRNAs, miRNAs can bind at the 5'UTR of target mRNAs and usually promote expression or translation of the target gene (da Sacco and Masotti, 2012). Identification of $B$. mori miRNAs that upregulate $B m F i b-L$ expression will be important in the study of the mechanisms of silk protein biosynthesis.

\section{Compliance with ethics guidelines}

Chen CHEN, Yang-yang FAN, Xin WANG, Fei SONG, Tao JIANG, Ping QIAN, Shun-ming TANG, and Xing-jia SHEN declare that they have no conflict of interest.

All institutional and national guidelines for the care and use of laboratory animals were followed.

\section{References}

Bushati, N., Cohen, S.M., 2007. MicroRNA functions. Annu. Rev. Cell Dev. Biol., 23(1):175-205. http://dx.doi.org/ 10.1146/annurev.cellbio.23.090506.123406

Cai, Y., Yu, X., Zhou, Q., et al., 2010. Novel microRNAs in silkworm (Bombyx mori). Funct. Integr. Genomics, 10(3): 405-415.

http://dx.doi.org/10.1007/s10142-010-0162-7

Cao, J., Tong, C., Wu, X., et al., 2008. Identification of conserved microRNAs in Bombyx mori (silkworm) and regulation of fibroin $\mathrm{L}$ chain production by microRNAs in heterologous system. Insect Biochem. Mol. Biol., 38(12): 1066-1071. http://dx.doi.org/10.1016/j.ibmb.2008.09.008

Chen, A., Xia, D., Qiu, Z., et al., 2013. Expression of a vitelline membrane protein, $B m V M P 23$, is repressed by bmo-miR-1a-3p in silkworm, Bombyx mori. FEBS Lett., 587(7):970-975. http://dx.doi.org/10.1016/j.febslet.2013.02.030

Couble, P., Moine, A., Garel, A., et al., 1983. Developmental variations of a nonfibroin mRNA of Bombyx mori 
silkgland, encoding for a low-molecular-weight silk protein. Dev. Biol., 97(2):398-407. http://dx.doi.org/10.1016/0012-1606(83)90096-9

da Sacco, L., Masotti, A., 2012. Recent insights and novel bioinformatics tools to understand the role of microRNAs binding to 5' untranslated region. Int. J. Mol. Sci., 14(1): 480-495. http://dx.doi.org/10.3390/ijms14010480

Dai, Z., Wu, R., Zhao, Y., et al., 2014. Early lethality of shRNA-transgenic pigs due to saturation of microRNA pathways. J. Zhejiang Univ.-Sci. B (Biomed. \& Biotechnol.), 15(5):466-473.

http://dx.doi.org/10.1631/jzus.B1400001

Ebert, M.S., Sharp, P.A., 2010. MicroRNA sponges: progress and possibilities. $R N A, \mathbf{1 6}(11): 2043-2050$. http://dx.doi.org/10.1261/rna.2414110

Fabian, M.R., Sonenberg, N., Filipowicz, W., 2010. Regulation of mRNA translation and stability by microRNAs. Annu. Rev. Biochem., 79(1):351-379.

http://dx.doi.org/10.1146/annurev-biochem-060308-103103

He, P.A., Nie, Z., Chen, J., et al., 2008. Identification and characteristics of microRNAs from Bombyx mori. BMC Genomics, 9(1):248. http://dx.doi.org/10.1186/1471-2164-9-248

Huang, Y., Zou, Q., Tang, S.M., et al., 2010. Computational identification and characteristics of novel microRNAs from the silkworm (Bombyx mori L.). Mol. Biol. Rep., 37(7):3171-3176. http://dx.doi.org/10.1007/s11033-009-9897-4

Huang, Y., Zou, Q., Wang, S.P., et al., 2011. Construction and detection of expression vectors of microRNA-9a in BmN cells. J. Zhejiang Univ.-Sci. B (Biomed. \& Biotechnol.), 12(7):527-533. http://dx.doi.org/10.1631/jzus.B1000296

Huang, Y., Zou, Q., Song, F., et al., 2012. The regulation of silkworm fibroin $\mathrm{L}$ chain production by miRNA-965 and miRNA-1926 in insect cells. Russ. J. Bioorg. Chem., 38(4):417-421.

http://dx.doi.org/10.1134/S1068162012030168

Inoue, S., Tanaka, K., Arisaka, F., et al., 2000. Silk fibroin of Bombyx mori is secreted, assembling a high molecular mass elementary unit consisting of H-chain, L-chain, and P25, with a 6:6:1 molar ratio. J. Biol. Chem., 275(51): 40517-40528. http://dx.doi.org/10.1074/jbc.M006897200

Johnston, R.J., Hobert, O., 2003. A microRNA controlling left/right neuronal asymmetry in Caenorhabditis elegans. Nature, 426(6968):845-849. http://dx.doi.org/10.1038/nature02255

Jopling, C.L., Yi, M., Lancaster, A.M., et al., 2005. Modulation of hepatitis $\mathrm{C}$ virus RNA abundance by a liver-specific microRNA. Science, 309(5740):1577-1581. http://dx.doi.org/10.1126/science.1113329

Khan, A.A., Betel, D., Miller, M.L., et al., 2009. Transfection of small RNAs globally perturbs gene regulation by endogenous microRNAs. Nat. Biotechnol., 27(6):549-555. http://dx.doi.org/10.1038/nbt.1543.

Kramer, M.F., 2011. Stem-loop RT-qPCR for miRNAs. Curr. Protoc. Mol. Biol., 15:15.10.1-15.10.15. http://dx.doi.org/10.1002/0471142727.mb1510s95

Lee, Y., Kim, M., Han, J., et al., 2004. MicroRNA genes are transcribed by RNA polymerase II. EMBO J., 23(20): 4051-4060. http://dx.doi.org/10.1038/sj.emboj.7600385

Li, J., Cai, Y., Ye, L., et al., 2014. MicroRNA expression profiling of the fifth-instar posterior silk gland of Bombyx mori. BMC Genomics, 15(1):410. http://dx.doi.org/10.1186/1471-2164-15-410

Lim, L.P., Glasner, M.E., Yekta, S., et al., 2003. Vertebrate microRNA genes. Science, 299(5612):1540. http://dx.doi.org/10.1126/science.1080372

Liu, S., Gao, S., Zhang, D., et al., 2010. MicroRNAs show diverse and dynamic expression patterns in multiple tissues of Bombyx mori. BMC Genomics, 11(1):85. http://dx.doi.org/10.1186/1471-2164-11-85

Liu, S., da Cunha, A.P., Rezende, R.M., et al., 2016. The host shapes the gut microbiota via fecal microRNA. Cell Host Microbe, 19(1):32-43. http://dx.doi.org/10.1016/j.chom.2015.12.005

Lu, Y.L., Jing, W., Feng, L.S., et al., 2014. Effects of hypoxic exercise training on microRNA expression and lipid metabolism in obese rat livers. J. Zhejiang Univ.-Sci. B (Biomed. \& Biotechnol.), 15(9):820-829. http://dx.doi.org/10.1631/jzus.B1400052

Mallory, A.C., Vaucheret, H., 2006. Functions of microRNAs and related small RNAs in plants. Nat. Genet., 38(7): 850-850. http://dx.doi.org/10.1038/ng1791

Poy, M.N., Eliasson, L., Krutzfeldt, J., et al., 2004. A pancreatic islet-specific microRNA regulates insulin secretion. Nature, 432(7014):226-230. http://dx.doi.org/10.1038/nature03076

Sen, R., Ghosal, S., Das, S., et al., 2014. Competing endogenous RNA: the key to posttranscriptional regulation. Sci. World J., 2014:896206. http://dx.doi.org/10.1155/2014/896206

Shalgi, R., Lieber, D., Oren, M., et al., 2007. Global and local architecture of the mammalian microRNA-transcription factor regulatory network. PLoS Comput. Biol., 3(7):e131. http://dx.doi.org/10.1371/journal.pcbi.0030131

Song, F., Huang, Y., Wang, X., et al., 2013. Bmo-miR-9a down regulates the expression of $\mathrm{Bm}$-ase gene in vitro. Russ. J. Bioorg. Chem., 39(2):170-175. http://dx.doi.org/10.1134/S1068162013020052

Song, F., Wang, X., Qian, P., et al., 2014. Expressional regulation of Bombyx mori fibroin heavy-chain gene by Bmo-miR-2739. Sci. Seric., 40(3):0404-0408. http://dx.doi.org/10.13441/j.cnki.cykx.2014.03.006

Song, F., Wang, X., Chen, C., et al., 2015. Characterization and profiling of microRNAs in posterior silk gland of the silkworm (Bombyx mori). Genes Genom., 37(8):703-712. http://dx.doi.org/10.1007/s13258-015-0300-7 
Sprague, K.U., 1975. Bombyx mori silk proteins: characterization of large polypeptides. Biochemistry, 14(5):925-931. http://dx.doi.org/10.1021/bi00676a008

Sun, X., Zhu, C., Wang, S., 2009. Quantitative analysis of fibroin genes' expression in silkworm, Bombyx mori. Sci. Seric., 35(1):30-35 (in Chinese). http://dx.doi.org/10.3969/j.issn.0257-4799.2009.01.006

Wang, G., Li, J., Xu, X., et al., 2011. Changes of fibroin gene expression during the different development period form silkworm. North Seric., 32(4):17-20 (in Chinese). http://dx.doi.org/10.3969/j.issn.1673-9922.2011.04.006

Wang, S., Lu, C., 2006. Leaked expression of cloned fibroin heavy chain promoter sequence in silkworm, Bombyx mori, and insect cultured cells. Sci. Seric., 32(4):491-494 (in Chinese). http://dx.doi.org/10.3969/j.issn.0257-4799.2006.04.008

Xia, Q., Zhou, Z., Lu, C., et al., 2004. A draft sequence for the genome of the domesticated silkworm (Bombyx mori). Science, 306(5703): 1937-1940. http://dx.doi.org/10.1126/science.1102210

Xia, Q., Guo, Y., Zhang, Z., et al., 2009. Complete resequencing of 40 genomes reveals domestication events and genes in silkworm (Bombyx). Science, 326(5951):433-436. http://dx.doi.org/10.1126/science.1176620

Yamaguchi, K., Kikuchi, Y., Takagi, T., et al., 1989. Primary structure of the silk fibroin light chain determined by cDNA sequencing and peptide analysis. J. Mol. Biol., 210(1):127-139. http://dx.doi.org/10.1016/0022-2836(89)90295-7

Yang, L., Lu, X., Liu, Y., et al., 2012. Expression analysis of miRNAs in BmN cells. Gene, 505(2):240-245. http://dx.doi.org/10.1016/j.gene.2012.06.018

Yu, X., Zhou, Q., Li, S.C., et al., 2008. The silkworm (Bombyx mori) microRNAs and their expressions in multiple developmental stages. PLoS ONE, 3(8):e2997. http://dx.doi.org/10.1371/journal.pone.0002997

Yu, X., Zhou, Q., Cai, Y., et al., 2009. A discovery of novel microRNAs in the silkworm (Bombyx mori) genome. Genomics, 94(6):438-444. http://dx.doi.org/10.1016/j.ygeno.2009.08.007

Yuan, Z.M., Yang, Z.L., Zheng, Q., 2014. Deregulation of microRNA expression in thyroid tumors. J. Zhejiang Univ.-Sci. B (Biomed. \& Biotechnol.), 15(3):212-224. http://dx.doi.org/10.1631/jzus.B1300192

Zeng, F., Xie, H., Nie, Z., et al., 2009. Characterization of the gene BmEm4, a homologue of Drosophila E(spl)m4, from the silkworm, Bombyx mori. Comp. Funct. Genomics, 2009:947490.

http://dx.doi.org/10.1155/2009/947490
Zhang, Y., Zhou, X., Ge, X., et al., 2009. Insect-specific microRNA involved in the development of the silkworm Bombyx mori. PLoS ONE, 4(3): 4677. http://dx.doi.org/10.1371/journal.pone.0004677

Zhao, Q.L., Shen, X.J., Zhu, L.J., et al., 2007. Characterization of CIb1 gene promoter from silkworm, Bombyx mori. Z. Naturforsch. C, 62(11-12):875-880.

Zhou, Y.J., Xiao, Q.L., Zhang, Z.F., et al., 2002. Foreign insect hormone stimulating the transcription of $i e-1$ promoter of Bombyx mori nuclear polyhedrosis virus in vivo and in vitro. Biosci. Biotechnol., Biochem., 66(7):1488-1494. http://dx.doi.org/10.1271/bbb.66.1488

\section{中文概 要}

\section{题 目: 家䖯 miR-0001 和 miR-0015 体外下调 BmFib-L 基因的表达}

目 的: 探究新发现的两个 miRNA 对家蚕丝素轻链基因 $B m F i b-L$ 的调控作用。

创新点: 在家虫后部丝腺中发现两个新的 miRNA, 并首次 证明它们对家蚕丝素蛋白轻链基因 BmFib- $L$ 有负 调控作用。

方 法: 本实验通过生物信息学分析, 从家蚕后部丝腺 miRNA 高通量测序获得的新 miRNA 中, 䇻选出 两个可能对家蚕丝素蛋白轻链基因 $B m F i b-L$ 有 调控作用的 miRNA, 即 bmo-miR-0001 和 bmomiR-0015。设计茎环引物, 采用反转录聚合酶链 反应 (RT-PCR) 方法对家蚕 5 龄 $3 \mathrm{~d}$ 头部、表皮、 脂肪体、马氏管、精巢/卵巢、丝腺（前、中、 后）、气管、中肠和血淋巴细胞等 12 个不同组 织的 bmo-miR-0001 和 bmo-miR-00015 进行半定 量表达分析。并采用双荧光报告基因检测系统进 一步在细胞水平上验证 bmo-miR-0001 和 bmo-miR-0015 对 BmFib- $L$ 表达的调控作用。

结 论: 本实验中 RT-PCR 结果显示, 这两个新 miRNA 在家蚕后部丝腺中表达量最高（图 4）。双荧光 报告基因检测结果显示，报告基因苂光素酶活性 明显低于阳性对照组(图 7), 转染 bmo-miR-0001 和 bmo-miR-0015 表达载体的细胞, 报告基因和 苂光素酶活性分别只及对照组 $60 \%$ 和 $69 \%$ 。 $t$ 检 验分析结果显示两个实验组与对照组之间差异 都达到显著水平 $(P<0.05)$ 。由此可见, bmo-miR-0001 和 bmo-miR-0015 在体外对 $B m F i b-L$ 的表达具有显著的抑制作用。

关键词：家虫; miRNA； bmo-miR-0001； bmo-miR-0015; $B m F i b-L$; 功能验证 